876

\section{DISORDERS OF THE BRAIN REVEALED BY MAGNETIC RESONANCE IMAGING (MRI) IN INFANTS WITH SEVERE CONGENITAL CYTOMEGALY}

B. Milewska-Bobula1 ${ }^{1}$, B. Lipka1, J. Zebrowska ${ }^{1}$, E. Jurkiewicz², I. Pakula-Kosciesza², D. Kuczynski ${ }^{3}$

${ }^{1}$ Department of Infant Diseases, ${ }^{2}$ Department of Imaging Diagnostics, ${ }^{3}$ Department of Neurology and Epileptology, The Children's Memorial Heath Institute, Warsaw, Poland

Aim: The aim of the study was to analyze the type of brain abnormalities in children with severe congenital cytomegaly, using MRI scan.

Material and methods: Seventeen infants congenitally infected by cytomegalovirus (CMV) hospitalized from 2002 to 2009 yrs were involved in the study. Congenital CMV infection was diagnosed on the basis of clinical signs and symptoms confirmed by detection of DNA CMV in urine and specific antibodies (IgM, IgG) in serum during the first weeks of life. In all MRI of the brain was performed. The mean period of observation was 17 months (ranged from 5 to 60 ).

Results: MRI findings: different types of malformations of cortical development (agyria, pachygyria, polimicrogyria, subependymal heterotopia) and focal cortical dysplasia in 17 $(100 \%)$, delayed myelinization in $6(35,3 \%)$, white matter lesions in $7(41,2 \%)$, thin corpus callosum in $5(30,0 \%)$, brain atrophy in $8(47,0 \%)$, cerebellar hypoplasia in $3(17,6 \%)$, ventriculomegaly in 15 $(88,2 \%)$, schizencephaly in $2(11,7 \%)$ infants. Other findings in CNS visualized by computer tomography: intracranial calcifications in $5(30,0 \%)$, microcephaly in 10 (60,0\%). Late sequelae: various degrees of psychomotor retardation $7(41,2 \%)$, epilepsy $8(47,0 \%)$, cerebral palsy $8(47,0 \%)$.

\section{Conclusions:}

1. Cortical dysplasia revealed in all infants as a result of cerebral migration defect indicate on fetal CMV infection during the first half of gestation.

2. Cortical malformations and other disorders of the brain were responsible for epilepsy, cerebral palsy and developmental delay.

3. MRI is the method of choice in evaluating brain lesions in children with cytomegaly.

\section{GROUP B STREPTOCOCCUS - FREQUENTLY RISK FACTOR FOR EARLY NEONATAL INFECTION}

\section{S.M. Stoicescu, D. Broscauncianu, C. Voicila}

NICU, Institute for Mother and Child Care - Polizu Hospital, Bucharest, Romania

Background: Neonatal infection is an important cause of neonatal mortality and morbidity. Group B Streptococcus agalactiae (GBS) during pregnancy and/or labor is one of the main risk factor for neonatal infection, which can be an early or a late, severe one.

Aim: To reduce neonatal morbidity and mortality

Objectives: To study maternal and neonatal GBS incidence,to establish the need for perinatal treatment,to reduce the newborn care costs.

Material and method: A retrospective study in Polizu Maternity between 2006-2009, in collaboration with "I. Cantacuzino" Institute for bacteriological serotyping. 14538 newborns were included in this study.

Results: GBS morbidity is raising from 2006 to 2009. One third of newborns from GBS mothers were also infected. About $1 \%$ of them were treated for a severe infection - sepsis.

Conclusions: The incidence of GBS infection in pregnant women and newborns is raising. Early diagnosis and treatment in perinatal period can explain the reduction of neonatal mortality.

\section{8}

\section{TLR2 AND TLR4 POLYMORPHISMS IN KAWASAKI DISEASE}

S. Greber-Platzer, E. Kitzmueller, K. Sadhegi, A. Dangl, K. Herkner, E. Förster-Waldl

Department of Paediatrics, Medical University of Vienna, Vienna, Austria

Kawasaki disease (KD) is a febrile acute systemic vasculitis in childhood, primarily affecting coronary arteries. Different causes are discussed including infectious agents, cofactors, and genetic predisposition. We tested the hypothesis that polymorphisms of TL-2 and TLR4, key mediators of the innate immune system influence coronary artery involvement in KD patients. 15 patients 